\title{
Mitochondrial dysfunction induced by callyspongiolide promotes autophagy-dependent cell death
}

\author{
Soohyun Lee ${ }^{1,2}$, Yoonjeong Jeong ${ }^{1}$, Jae-Seok Roe ${ }^{2}$, Hoyoung Huh ${ }^{1}$, Sang Hoon Paik ${ }^{1, *}$ E Jaewhan Song ${ }^{1,2, *}$ \\ ${ }^{1} \mathrm{pH}$ Pharma Co., Ltd., Seongnam 13494, ${ }^{2}$ Department of Biochemistry, College of Life Science and Biotechnology, Yonsei University, Seoul \\ 03722, Korea
}

\begin{abstract}
Callyspongiolide is a marine macrolide known to induce caspaseindependent cancer cell death. While its toxic effects have been known, the mechanism leading to cell death is yet to be identified. We report that Callyspongiolide $R$ form at C-21 (cally2R) causes mitochondrial dysfunction by inhibiting mitochondrial complex I or II, leading to a disruption of mitochondrial membrane potential and a deprivation of cellular energy. Subsequently, we observed, using electron microscopy, a drastic formation of autophagosome and mitophagy. Supporting these data, LC3, an autophagosome marker, was shown to co-localize with LAMP2, a lysosomal protein, showing autolysosome formation. RNA sequencing results indicated the induction of hypoxia and blocking of EGF-dependent pathways, which could be caused by induction of autophagy. Furthermore, mTOR and AKT pathways preventing autophagy were repressed while AMPK was upregulated, supporting autophagosome progress. Finally, the combination of cally2R with known anti-cancer drugs, such as gefitinib, sorafenib, and rapamycin, led to synergistic cell death, implicating potential therapeutic applications of callyspongiolide for future treatments. [BMB Reports 2021; 54(4): 227-232]
\end{abstract}

\section{INTRODUCTION}

Autophagy is an essential biological process for the degradation and elimination of damaged proteins and cellular components. It plays a significant role in maintaining cellular homeostasis under conditions of starvation, low ATP levels, hypoxia, and endoplasmic reticulum stress (1-3). In cancer biology, autophagy

*Corresponding authors. Jaewhan Song, Tel: +82-31-779-5302; Fax: +82-31-779-5329; E-mail: jso678@yonsei.ac.kr; Sang Hoon Paik, Tel: +82-31-779-5302; Fax: +82-31-779-5329; E-mail: sanghoon.paik@ ph-pharma.com

https://doi.org/10.5483/BMBRep.2021.54.4.037

Received 5 March 2021, Revised 17 March 2021, Accepted 1 April 2021

Keywords: Autophagy, Callyspongiolide, Metabolism, Mitochondria, Mitophagy could play dual roles in the process of tumor suppression and promotion. Autophagy-regulated chemotherapy can be involved in either cancer cell survival or death. However, in tumors, excessive autophagy can result in apoptosis and cell death, consequentially leading to death of drug-resistant tumor cells $(4,5)$. Cancer metabolism usually refers to the alterations in cellular metabolism pathways, which includes changes in aerobic glycolysis, oxidative phosphorylation and the generation of biosynthetic intermediates needed for cell growth and proliferation $(6,7)$. Numerous fuel sources for the synthesis of macromolecules could be achieved via aerobic glycolysis. Also, mitochondrial metabolism has been demonstrated to be critical for ATP production, a redox balance, and the biosynthesis of other key metabolites in various tumor types. However, under energy-deprived conditions, cancer cells can activate the autophagy pathway with the suppression of mTOR signals to prohibit cell growth (8). In addition, AMP-regulated kinase (AMPK) promotes autophagy through phosphorylation of ULK kinases, which is required for cell survival during starvation $(9$, 10). AMPK signaling activated during low energy conditions is also involved in direct inhibition of multiple targets, including mTORC1, to restore energy homeostasis (9). AMPK activation in response to electron transport chain dysfunction results in mitochondrial fragmentation through direct phosphorylation of mitochondrial fission factor, an OMM receptor for Drp1 (11). Finally, sustained energy deprivation can result in AMPKmediated upregulation of mitochondrial biogenesis.

Many macrolides show potent anti-proliferative properties against cancer cells, making them promising leads to develop new chemotherapeutics agents. One of the macrolides, callyspongiolide, is a marine-derived macrolide natural product that displays caspase-independent cytotoxic activity (12). This implies a non-apoptotic mechanism for cell death $(13,14)$. Because apoptotic signaling is suppressed in many cancers, often underpinning drug resistance in clinical settings, compounds that kill cancer cells by non-apoptotic mechanisms are valuable to explore as plausible therapeutic tools $(14,15)$.

In this paper, we focused on the induction of cell death induced by $\mathrm{R}$ form of callyspongiolide (cally2R), one of the two callyspongiolide isoforms ( $R$ and $S$ forms at $C-21)$. Cally $2 R$ causes cellular energy deprivation by inducing mitochondrial dysfunction, resulting in autophagy-dependent cell death. Com-

ISSN: 1976-670X (electronic edition)

Copyright (C) 2021 by the The Korean Society for Biochemistry and Molecular Biology

(c) This is an open-access article distributed under the terms of the Creative Commons Attribution Non-Commercial License (http://creativecommons.org/licenses/by-nc/4.0) which permits unrestricted non-commercial use, distribution, and reproduction in any medium, provided the original work is properly cited. 
binational treatment of known drugs with cally2R displayed potent effects on cell death, promising possible therapeutic application in the future. We conclude that cally2R could be an effective anti-cancer drug for breast cancer.

\section{RESULTS}

The $\mathbf{R}$ form of callyspongiolide shows anti-cancer activities in various cancer cell lines at a nanomolar range

To investigate the pharmacological potential of callyspongiolide, the toxicity of callyspongiolide isoforms at C-21 (Fig. 1A) was tested on various cancer cells over 72 hours at a range of concentrations $(0.1-10,000 \mathrm{nM})$. The results showed that cally $2 R$ had higher potency than the $S$ form and induced cell death at nanomolar ranges (Fig. 1B). The half-maximal inhibitory concentrations $\left(\mathrm{IC}_{50}\right)$ of cally2R on SKOV3, N87, SKBR3, HT29, BT474, HPAFII, SW480, MCF7, HCT116, A549, HepG2, and 786-O were $10.3 \mathrm{nM}, 15.9 \mathrm{nM}, 25.3 \mathrm{nM}, 5.7$ $\mathrm{nM}, 21.5 \mathrm{nM}, 7.1 \mathrm{nM}, 16.4 \mathrm{nM}, 11.1 \mathrm{nM}, 9.6 \mathrm{nM}, 5.3 \mathrm{nM}$, $7.3 \mathrm{nM}$ and $10.0 \mathrm{nM}$, respectively (Fig. 1B). Z-vad-fmk (pancaspase inhibitor), GSK963 (RIPK1 inhibitor; Necroptosis inhibitor), or Ferrostatin-1 (Ferroptosis inhibitor) were treated on cells in the presence of cally $2 R$ to elucidate how cally $2 R$ induces cell death. All the tested inhibitors failed to suppress cell death induced by cally $2 R$. This indicates that cally $2 R$

A

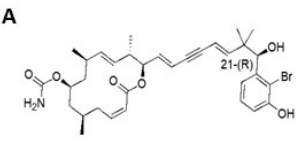

Callyspongiolide (R)

B

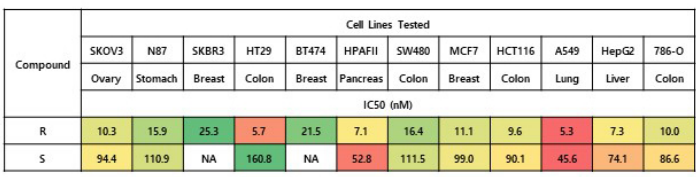

C

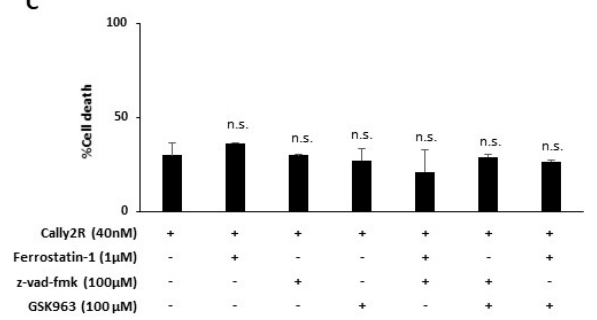

Fig. 1. Various cancer cell lines undergo cell death under the treatment of cally $2 R$. (A) A structure of callyspongiolide $R$ and $S$ form at $\mathrm{C}-21$. (B) $\mathrm{IC}_{50}$ of cally $2 \mathrm{R}$ after $72 \mathrm{~h}$ of cally2R treatment in various cell lines $(n=3)$. (C) CTG assay in SKBR3 cells were treated of cally2R, ferrostatin-1, z-VAD-fmk or GSK963 for $48 \mathrm{~h}$ $(n=3)$. Each bar represents the mean + s.d. n.s. no significance; $* \mathrm{P}<0.05, * * \mathrm{P}<0.01, * * * \mathrm{P}<0.001$ compared to the cally $2 \mathrm{R}-$ treated group. generates cell death independent of caspase, necroptosis and ferroptosis (Fig. 1C). The results suggest that cally2R induces specific toxicity on cancer cells independent of apoptosis, necroptosis, or ferroptosis.

\section{Cally2R triggers the formation of autolysosomes in breast cancer cells}

Among various cell lines tested, we focused on breast cancer to assess the mechanism of cally2R. First, to identify the type of cell death induced by cally $2 \mathrm{R}$ on breast cancer cell lines, the morphologies of SKBR3 and MDA-MB-453 treated with cally2R were visualized using a transmission electron microscope (TEM). Consistent with the observations that cally $2 \mathrm{R}$ acts on cell death pathway independent of apoptosis or necrosis, no distinguishing feature of cell death types such as cell shrinkage, blebbing, or plasma membrane rupture with swelling were observed. Interestingly, vast numbers of autophagic vacuoles appeared under cally2R treatment (Fig. 2A). We observed the appearance of autophagosomes, which are early autophagic vacuoles (red arrows), and autolysosomes, which are late autophagic vacuoles (blue arrows), in cells treated with cally $2 \mathrm{R}$. Also, the process of mitophagy was observed, indicated by yellow arrows (Fig. 2A). As a control, cells treated with metformin, which is known to induce autophagy, were compared with cells treated with cally $2 R$ (16). Only limited numbers of small-sized autophagosomes were observed using metformin as previously reported (16). This suggests that cally2R has more potent autophagic inducing effects on cancer cells than metformin (Fig. 2A). The effects of cally2R on the formation of autophagosome were further analyzed in SKBR3 and HT29 cells. Both cell lines displayed (I) formation of a cup-shaped membrane structure enclosing a portion of the cytoplasm, (II) visible mature autophagic bodies, and (III) autophagic bodies in the final stage with most cytoplasmic materials degraded (Fig. 2B). The colocalization of LC3 (green), an autophagosome marker, and LAMP2 (red), a lysosome marker, further confirms the effects of cally2R on the induction of autolysosome (Fig. 2C). In addition, we showed with western blots that there was the conversion of LC3 from LC-1 to LC3-II under cally $2 \mathrm{R}$ treatment. This indicates an increase of autophagosome formation in breast cancer cells in a time-dependent manner. Of note, the levels of p62, which is incorporated into complete autophagosomes and is degraded by lysosomes (17), was maintained with cally2R treatment (Fig. 2C). This might be due to cancer cells going through the production of continuous autophagosomes without disruption of these processes under cally2R treatment. In addition to the changes of direct autophagic factors, we observed the hallmarks of autophagic processes with decreased $\mathrm{p}-\mathrm{mTOR}$ and $\mathrm{p}-4 \mathrm{E}-\mathrm{BP}$ and increased protein levels of $\mathrm{p}$-AMPK under cally2R treatment (Fig. 2D). Moreover, proteins related to mitophagy, such as BNIP3, NDP52, and $\mathrm{PINK}$, increased with cally2R treatment, and the results are corresponding to the TEM data shown above (Fig. 2A, D). These results demonstrate that cally $2 \mathrm{R}$ can induce autophagosome 
A
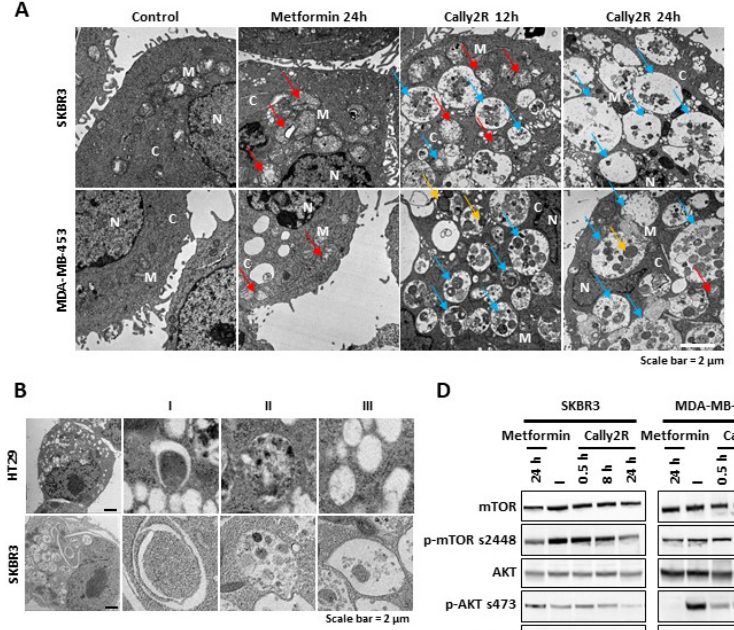

c

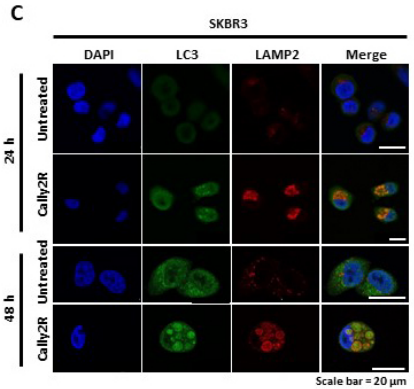

D

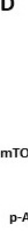

кквR3

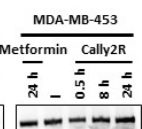

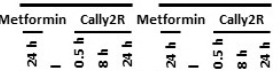

10

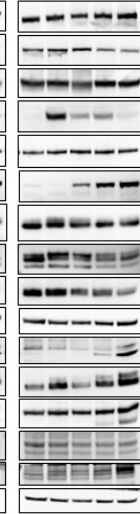

Fig. 2. Cally $2 R$ treatment induces autolysosome fusion with suppression of the mTOR/AKT pathway and activation of AMPK. (A) The typical TEM images of autophagic vacuoles in SKBR3 and MDAMB-453 cells after treatment of $3 \mathrm{mM}$ metformin or $100 \mathrm{nM}$ cally2R. Scale bar $=2 \mu \mathrm{m}$. (B) TEM images of the three stages of autophagic vacuoles in colon (HT29) and breast (SKBR3) cancer cells after cally2R treatment for $24 \mathrm{~h}$. Stage (I): A cup-shaped membrane structure enclosing a portion of cytoplasm. Stage (II): Visible autophagic bodies in the vacuole. Stage (III): Autophagic bodies in the vacuole, showing degradation of the segregated cytoplasm. Scale bar $=2 \mu \mathrm{m}$. (C) Immunocytochemistry analysis by staining with anti-LC3 antibody (green) and anti-LAMP2 antibody (red) shows the formation of autolysosomes (merged as yellow) after cally2R treatment in SKBR3 cells. Nuclei were counterstained with DAPI (blue). Scale bar $=20 \mu \mathrm{m}$. (D) Immunoblotting showing protein levels of mTOR pathway and mitophagy after cally2R treatment. $\mathrm{C}$, cytosol; $\mathrm{M}$, mitochondria; $\mathrm{N}$, nucleus; yellow arrow, mitochondria in autophagosome; red arrow, autophagosomes; blue arrow, autolysosomes.

accumulation and autophagosome-lysosome fusion. In addition, the signaling pathways stimulating autophagosome and mitophagy were activated, while the opposing pathways were downregulated in breast cancer cells.

\section{Cally2R induces mitochondrial dysfunction causing energy deprivation and cell cycle arrest}

What would be the cause of vast autolysosome appearances under cally2R treatment? Since we observed significant amounts

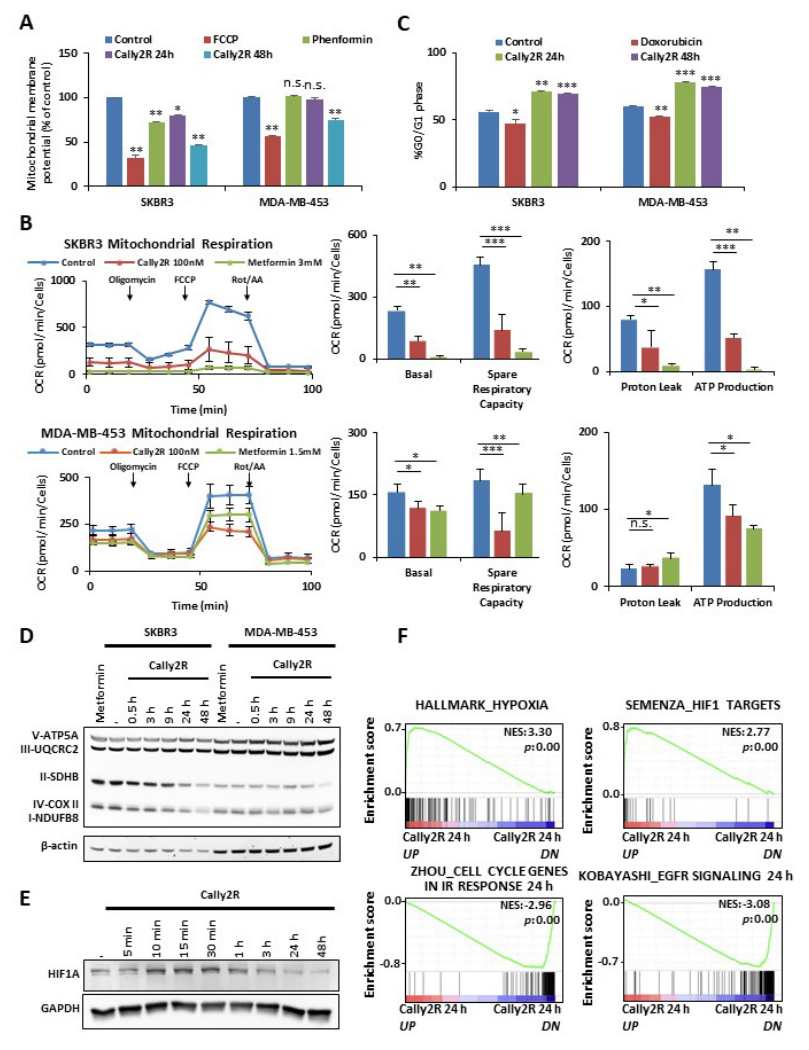

Fig. 3. Cally $2 R$ induces mitochondrial dysfunction causing energy deprivation and cell cycle arrest. (A) TMRE assay showing that cally2R reduces membrane potential in SKBR3 and MDA-MB-453 cells ( $n$ $=3$ ). $20 \mu \mathrm{M}$ FCCP was used as a positive control. (B) Cell cycle analysis showing that cally2R induces G0/G1 phase arrest in $24 \mathrm{~h}$ at $100 \mathrm{nM}(\mathrm{n}=3)$. $1 \mu \mathrm{M}$ doxorubicin was used as a positive control. (C) Mito stress test shows that cally2R reduces OCR and respiration parameters in SKBR3 and MDA-MB-453 cells in $24 \mathrm{~h}$ $(n=3)$. Immunoblotting showing protein levels of (D) mitochondrial complexes in SKBR3 and MDA-MB-453 cells and (E) HIF1A in SKBR3 cells after $3 \mathrm{mM}$ metformin or $100 \mathrm{nM}$ cally2R treatment. (F) Gene set enrichment analysis (GSEA) of RNA sequencing after $100 \mathrm{nM}$ cally2R treatment in SKBR3 cells. GSEA analysis showed that Hypoxia and Hif1 target genes are upregulated, and cell cycle, EGFR signaling are downregulated after cally2R treatment. Each bar represents the mean + s.d. n.s., no significance; $* P<0.05, * * P<$ $0.01, * * * \mathrm{P}<0.001$.

of mitochondria taken up by autophagosomes, the mitochondrial functional integrity was further examined. First, the mitochondrial membrane potential was tested using Tetramethylrhodamine ester perchlorate (TMRE). The mitochondrial membrane potential was reduced by $20 \%$ and $53 \%$ in SKBR3 and $2 \%$ and $26 \%$ in MDA-MB-453 cells when treated with Cally $2 \mathrm{R}$ for 24 and $48 \mathrm{~h}$, respectively (Fig. 3A). Since the mitochondrial membrane potential is disrupted under cally $2 \mathrm{R}$ treatment, we subsequently tested oxygen consumption rates (OCR) under similar conditions.

The results showed that all respiratory parameters, including 
basal respiration, spare respiratory capacity, proton leak, and ATP production were significantly reduced in SKBR3 cells when treated with cally2R or metformin (control). Similarly, there was a reduction in the spare respiratory capacity, basal respiration, and ATP production in MDA-MB-453 cells but not in proton leak. (Fig. 3B). It is known that the reduction in mitochondrial activity has an effect on cell cycle efficiency in cancer cells (18). Therefore, we analyzed the cell cycle in SKBR3 and MDA-MB-453 cells. The data showed that cally2R treatment induces cell cycle arrest in the G0/G1 phase (Fig. $3 C)$, suggesting that cally2R-induced ATP depletion affects cell cycle arrest in breast cancer cells. Next, western blots were performed to analyze mitochondrial complexes in breast cancer cells after cally2R treatment. Protein levels of mitochondrial complexes I and II were decreased in a time-dependent manner with cally $2 \mathrm{R}$ treatment, suggesting toxic effects of cally $2 \mathrm{R}$ on reducing $\mathrm{NADH}$ and $\mathrm{FADH}_{2}$ (19). There were no significant changes to the levels of complexes III, IV, and V when treated with cally2R (Fig. 3D). We further tested whether cally $2 R$ could induce hypoxia, because studies have shown that mitochondrial dysfunction and ATP deprivation induces hypoxia (20). As expected, Hypoxia Inducible Factor 1 Subunit Alpha (HIF1A) was increased within 10 min of cally2R treatment, suggesting that cally $2 \mathrm{R}$ could induce hypoxia in breast cancer cells. Lastly, the RNA sequencing analysis showed that cally2R treatment in SKBR3 cells could induce hypoxia-related genes and hif1 target genes, but reduce cell cycle associated genes (Fig. 3F). Following mTOR data shown in Fig. 2C, genes associated with EGFR signaling pathways were also downregulated (Fig. 3F). Overall, the data suggests that cally2R could induce mitochondrial dysfunction with energy deprivation, resulting in the induction of hypoxia pathway with the suppression of cell growth signaling, including cell cycle and EGFR pathways.

\section{Combined treatment of cally2R with growth inhibitors led to synergistic induction of cell death in breast cancer cells}

Chemicals affecting cancer cell metabolism can have more destructive effects on cell death when co-treated with cell growth inhibitors $(3,21)$. Therefore, we tested whether cally $2 R$ could induce more deleterious results when combined with cell growth inhibitors such as gefitinib, sorafenib, or rapamycin. There was a significant increase in cell death when cally $2 R$ was treated in combination with sorafenib, gefitinib, or rapamycin in SKBR3 and MDA-MB-453 cells (Fig. 4A, B). Metformin and phenformin were used to compare the combined effect with cally $2 R$. The combination treatment of cally $2 R$ with growth inhibitors was much effective than the combination treatment with metformin or phenformin (Fig. 1A). Since cally2R could induce a drastic formation of autophagosomes and autolysosomes, we further tested whether serum deprivation could accelerate cell death. As expected, there was a significant cell death increase in serum-starved SKBR3 and MDA-MB-453 cells with treatment of cally2R (Fig. 4C). Metformin, a positive control,

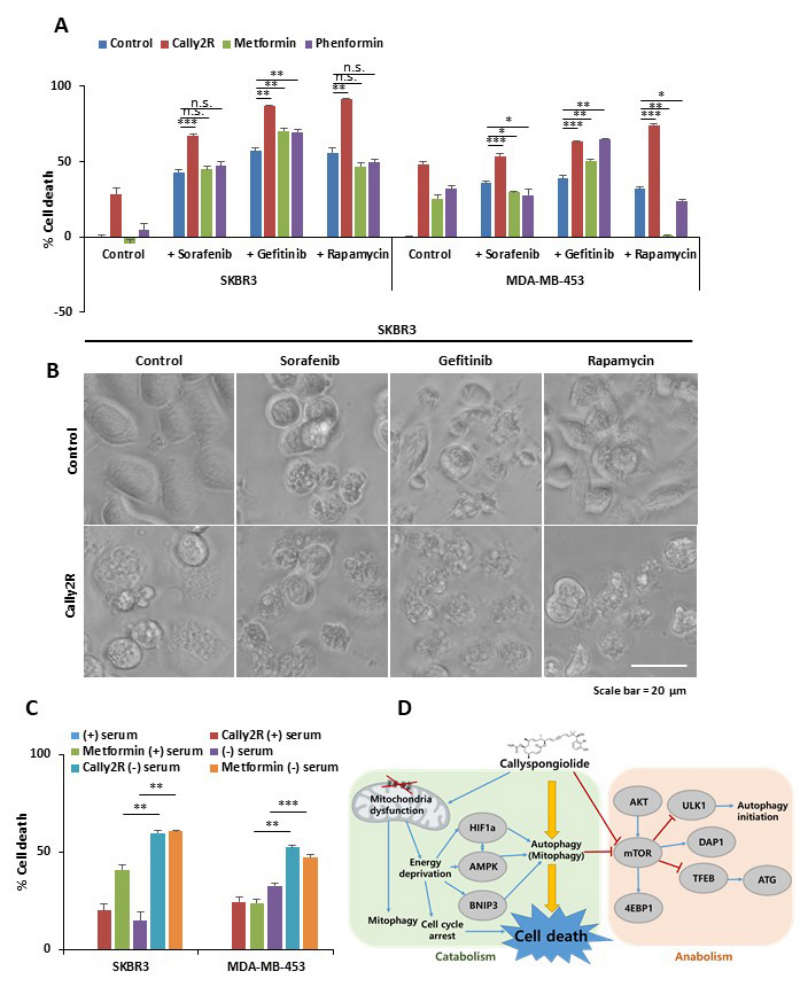

Fig. 4. Combined treatment of cally $2 R$ with growth inhibitors led to synergistic induction of cell death. (A) CTG assay showing the synergistic effect of cally $2 \mathrm{R}$ and growth inhibitors, $20 \mu \mathrm{M}$ sorafenib, $20 \mu \mathrm{M}$ gefitinib, or $20 \mu \mathrm{M}$ rapamycin, on cell death in SKBR3 and MDA-MB-453 cells after $48 \mathrm{~h}(\mathrm{n}=3)$. (B) SKBR3 cells were treated with cally $2 \mathrm{R}$ combined with sorafenib, gefitinib, or rapamycin for $48 \mathrm{~h}$, the cells were imaged through a microscope. Scale bar = $20 \mu \mathrm{m}$. (C) CTG assay showing the synergistic effect of cally $2 \mathrm{R}$ and serum starvation on cell death in SKBR3 and MDA-MB-453 cells after $48 \mathrm{~h}(\mathrm{n}=3)$. (D) A proposed model for the mechanism of cally $2 \mathrm{R}$ underlying inhibition of mitochondria and $\mathrm{mTOR}$ pathway in breast cancer. Each bar represents the mean + s.d. n.s., no significance; $* P<0.05, * * P<0.01, * * * P<0.001$.

showed similar effects. Overall, these data suggest that cally2R could have therapeutic potential particularly when applied with known anti-cancer drugs.

\section{DISCUSSION}

The effects of cally $2 \mathrm{R}$ on cancer cells are very peculiar in that it induces large numbers of autophagosomes without the dissolution of these vacuoles. Under normal starved conditions, it is known that the autophagic vacuoles, once energy homeostasis is achieved, are dissolved possibly via mTOR activation. Under cally2R treatment, there was continuous repression of mTOR pathway with activation of signaling pathways including AMPK, mitophagy, and autophagy, which might inhibit autophagic vacuoles eventual disappearances. The TEM data clearly showed the morphology of early autophagosomes, mitophagic vacuoles, 
and late autolysosomes, which was also detected with colocalization of LC3 and LAMP2. We speculate that the eruption of these vacuoles is due to the dysfunction of mitochondria under the presence of cally $2 \mathrm{R}$. It seems that cally $2 \mathrm{R}$ could have a negative effect on complex I and II because there was a decrease of the components of these complexes under cally $2 R$ treatment. This could lead to disruption of mitochondrial membrane potential and energy deprivation which could instantly cause energy deprivation. These processes seem to initiate AMPK activation, HIF1A stabilization and BNIP3 induction, all of which could be involved in the induction of autophagy as well as mitophagy. Under these conditions, mTOR pathways are completely blocked leading to cell cycle and growth arrest. Eventually, without recuperation of mitochondria, cellular homeostasis' irreversible disruption would occur, leading to cell death (Fig. 4D). It did not escape our notice that cally2R might affect ATP synthase as it is known that cally2R could suppress vacuolar ATPase of yeast, which have somewhat similar complex structures of ATP synthase of mitochondria $(22,23)$. Of note, there could be promising therapeutic effects of cally $2 \mathrm{R}$ in the future. First of all, the $\mathrm{IC}_{50}$ range for cally $2 \mathrm{R}$ is approximately a few $\mathrm{nM}$ depending on the cancer cell types, which might enable its application for antibody-drug conjugates. Secondly, this compound's combinational treatment could multiply the effects of other drugs, especially inducing cell growth arrest and death. Further experiments to identify analogs of cally $2 \mathrm{R}$ with more potent therapeutic effects are needed in future studies.

\section{MATERIALS AND METHODS}

\section{Reagents}

$\mathrm{pH}$ Pharma acquired an exclusive license from Purdue Research Foundation and synthesized Callyspongiolide at Chemveda. The primary antibodies of mTOR, p-mTOR s2448, AMPK, p-AMPK, AKT, p-AKT, p-4E-BP t37/46, p-4E-BP t70, 4E-BP, BNIP3, NDP52, parkin, HIF1A, and GAPDH were purchased from Cell Signaling Technology. The primary antibodies of p62 were purchased from BD Biosciences, LC3 from Medical \& Biological Laboratories, OXPHOS cocktail, and LAMP2 from Abcam, and $\beta$-actin from Santa Cruz Biotechnology.

\section{Cell culture}

SKBR3, MDA-MB-453, HT29, MCF7, HCT116, N87, and BT474 cells were purchased from the Korean Cell Line Bank. All cells were grown in RPMI 1640 medium (HyClone) containing 10\% fetal bovine serum (HyClone), penicillin, and streptomycin (HyClone). Cells were incubated at $37^{\circ} \mathrm{C}$ and maintained at $5 \% \mathrm{CO}_{2}$.

\section{TEM}

Cells were treated with Cally2R for the indicated time. Cells were then fixed with $2.5 \%$ glutaraldehyde (Sigma) in $0.1 \mathrm{M}$ phosphate $(\mathrm{pH}$ 7.4). The samples were placed on grids and subsequently captured results using a LIBRA 120 EF-TEM (Carl
Zeiss) with the help from SNU to prepare the samples for TEM imaging at the SNU microscopy facility.

\section{Cell Titer-Glo (CTG) assay}

Cells were incubated with callys $2 \mathrm{R}$ and indicated inhibitors for indicated times. After incubation, treated cells were imaged by a microscope and analyzed by CTG Luminescent Cell Viability Assay (Promega) according to the manufacturer's instructions. $\%$ Cell death was determined by following calculation: \%Cell death $=(1-$ ATP sample/ATP control $) \times 100$.

\section{Immunocytochemistry}

Cells were seeded on glass coverslips and were treated with Cally2R for the indicated time. Cells were fixed with $4 \%$ paraformaldehyde (Sigma) for $20 \mathrm{~min}$ at RT and then permeabilized with $0.1 \%$ Triton-X 100 (Sigma) for $20 \mathrm{~min}$ at $4^{\circ} \mathrm{C}$. After blocking with $3 \%$ BSA in PBS, cells were stained overnight at $4^{\circ} \mathrm{C}$ with LC3 and LAMP2 antibody, followed by Alexa Fluor 555-conjugated anti-mouse and Alexa Fluor 488conjugated anti-rabbit (Invitrogen) secondary antibodies diluted in $3 \%$ BSA/PBS for $1 \mathrm{~h}$ at RT in the dark. Cells were then mounted with 4',6-diamidino-2-phenylindole (DAPI) mounting medium (VECTASHIELD) to visualize nuclei. Samples were examined under Olympus confocal microscope.

\section{Western blot analysis}

Cells were treated with Callys2R or indicated inhibitors for the indicated time. Harvested cells were lysed with RIPA buffer in the presence of protease and phosphatase inhibitor cocktail (Biorad). The protein concentration of the cell lysates was quantified by a BCA Protein Assay Kit (Invitrogen). The same amount of protein samples was loaded onto 4-15\% SDS-PAGE and transferred onto PVDF membranes. After blocking by $5 \%$ BSA in TBST, the membranes were incubated in the primary antibodies diluted in $5 \%$ BSA buffer overnight at $4^{\circ} \mathrm{C}$ and then in the HRP-conjugated secondary antibody (Invitrogen) for $1 \mathrm{~h}$ at RT. The protein band images were captured with an ECL reagent (Biorad).

\section{Cell cycle assay}

Cells were treated with indicated inhibitors for indicated time. Cells were fixed with $70 \%$ ethanol by adding drop by drop to the cell pellet and kept at $4^{\circ} \mathrm{C}$ overnight. Cells were washed and resuspended with $0.5 \mathrm{ml}$ of FxCycle ${ }^{\mathrm{TM}} \mathrm{PI} / \mathrm{RNAse}$ Solution (Invitrogen), incubated for $30 \mathrm{~min}$, and analyzed by flow cytometry.

\section{OCR analysis}

XF Cell Mito Stress kit (Seahorse Bioscience) was used to determine OCR, and the experiment was done according to the manufacturer's instructions. Cells were seeded in XFe24 cell culture microplate and treated with cally $2 \mathrm{R}$ or metformin (Sigma) for $48 \mathrm{~h}$ at $37^{\circ} \mathrm{C}$. Cells were incubated in an XF base medium supplemented with $10 \mathrm{mM}$ glucose and $2 \mathrm{mM}$ 
L-glutamine and equilibrated in a non- $\mathrm{CO}_{2}$ incubator for $1 \mathrm{~h}$ before starting the assay. The samples were mixed ( $3 \mathrm{~min}$ ) and measured (3 min) using XFe24 extracellular flux analyzer. 2.5 $\mu \mathrm{M}$ Oligomycin, $1 \mu \mathrm{M}$ FCCP, and $0.5 \mu \mathrm{M}$ Rotenone/Antimycin A were injected at the indicated time points. The experiment was normalized by SRB assay.

\section{Measurement of mitochondrial membrane potential}

TMRE-Mitochondrial Membrane Potential Assay Kit (Abcam) was used to measure mitochondrial membrane potential, and the experiment was done according to the manufacturer's instructions. Cells were treated with indicated inhibitors for the indicated time. $200 \mathrm{nM}$ TMRE was added to the culture medium and incubated for $30 \mathrm{~min}$ at $37^{\circ} \mathrm{C}$. Cells were harvested, resuspended with $0.5 \mathrm{ml} \mathrm{PBS}$ and were analyzed by flow cytometry.

\section{Sulforhodamine B (SRB) assay}

Cells were fixed by adding 33\% TCA and kept for $1 \mathrm{~h}$ at $4^{\circ} \mathrm{C}$. After washing with tap water, $0.4 \%$ SRB (Sigma) solution in $1 \%$ acetic acid (Sigma) was added to each well, and the plates were kept for $5 \mathrm{~min}$ at RT. After staining, the plates were washed with $1 \%$ acetic acid and air-dried. The bound stain was solubilized with $1 \%$ Trizma buffer (Sigma), and the absorbance of the dye in solution is measured at OD $515 \mathrm{~nm}$.

\section{Statistical analysis}

Statistical analysis was performed in Microsoft Excel using the Student's t-test with a $\mathrm{P}$ value of $<0.05$ as the basis for rejection of the null hypothesis.

\section{ACKNOWLEDGEMENTS}

The authors are grateful for the use of Seoul National University's imaging facility and we thank the technicians for technical support. We are grateful to Drs. Sammy C.S. Lee and Myeong Jin Yoon for their help with critical reading and improving the manuscript.

\section{CONFLICTS OF INTEREST}

The authors have no conflicting interests.

\section{REFERENCES}

1. Linder B, Kogel D (2019) Autophagy in cancer cell death. Biology (Basel) 8, 82

2. Akkoc Y, Gozuacik D (2018) Autophagy and liver cancer. Turk J Gastroenterol 29, 270-282

3. Kim SY, Hwangbo H, Kim MY et al (2021) Coptisine induces autophagic cell death through down-regulation of $\mathrm{PI} 3 \mathrm{~K} / \mathrm{Akt} / \mathrm{mTOR}$ signaling pathway and up-regulation of ROS-mediated mitochondrial dysfunction in hepatocellular carcinoma Hep3B cells. Arch Biochem Biophys 697, 108688

4. Sanchez-Alvarez M, Strippoli R, Donadelli M, Bazhin AV, Cordani M (2019) Sestrins as a therapeutic bridge between ROS and autophagy in cancer. Cancers (Basel) 11, 1415
5. Bao J, Liu B, Wu C (2020) The prospects of therapeutic potential and drug development targeting autophagy in cancer. Adv Exp Med Biol 1207, 663-679

6. Nyfeler B, Eng CH (2016) Revisiting autophagy addiction of tumor cells. Autophagy 12, 1206-1207

7. Vander Heiden MG, Cantley LC, Thompson CB (2009) Understanding the Warburg effect: the metabolic requirements of cell proliferation. Science 324, 1029-1033

8. Bernardi P (1999) Mitochondrial transport of cations: channels, exchangers, and permeability transition. Physiol Rev 79, 1127-1155

9. Faubert B, Vincent EE, Poffenberger MC, Jones RG (2015) The AMP-activated protein kinase (AMPK) and cancer: many faces of a metabolic regulator. Cancer Lett 356, 165-170

10. Vyas S, Zaganjor E, Haigis MC (2016) Mitochondria and cancer. Cell 166, 555-566

11. Toyama EQ, Herzig S, Courchet J et al (2016) Metabolism. AMP-activated protein kinase mediates mitochondrial fission in response to energy stress. Science 351, 275-281

12. Pham CD, Hartmann R, Bohler P et al (2014) Callyspongiolide, a cytotoxic macrolide from the marine sponge Callyspongia sp. Org Lett 16, 266-269

13. Pommier $Y$, Sordet $\mathrm{O}$, Antony S, Hayward RL, Kohn KW (2004) Apoptosis defects and chemotherapy resistance: molecular interaction maps and networks. Oncogene 23, 2934-2949

14. Chang YH, Yang YL, Chen CM, Chen HY (2015) Apoptosis pathway signature for prediction of treatment response and clinical outcome in childhood high risk B-Precursor acute lymphoblastic leukemia. Am J Cancer Res 5, 1844-1853

15. Manoni F, Rumo C, Li L, Harran PG (2018) Unconventional fragment usage enables a concise total synthesis of (-)-callyspongiolide. J Am Chem Soc 140, 1280-1284

16. Wang Y, Xu W, Yan Z et al (2018) Metformin induces autophagy and G0/G1 phase cell cycle arrest in myeloma by targeting the AMPK/mTORC1 and mTORC2 pathways. J Exp Clin Cancer Res 37, 63

17. Bjorkoy G, Lamark T, Pankiv S, Overvatn A, Brech A, Johansen T (2009) Monitoring autophagic degradation of p62/SQSTM1. Methods Enzymol 452, 181-197

18. Fernandez-Marcos PJ, Serrano M (2016) Mitochondrial damage induces senescence with a twisted arm. Cell Metab 23, 229-230

19. Ahmad M, Wolberg A, Kahwaji Cl (2021) Biochemistry, electron transport chain. StatPearls Publishing; 2021 Jan. 2020 Sep 8

20. Agani FH, Pichiule P, Chavez JC, LaManna JC (2000) The role of mitochondria in the regulation of hypoxia-inducible factor 1 expression during hypoxia. J Biol Chem 275, 3586335867

21. Huang Y, Zhou S, He C et al (2018) Phenformin alone or combined with gefitinib inhibits bladder cancer via AMPK and EGFR pathways. Cancer Commun (Lond) 38, 50

22. Fogarty S, Ouyang Y, Li L et al (2020) Callyspongiolide Is a potent inhibitor of the vacuolar ATPase. J Nat Prod 83, 3381-3386

23. Fethiere J, Venzke D, Diepholz M et al (2004) Building the stator of the yeast vacuolar-ATPase: specific interaction between subunits E and G. J Biol Chem 279, 40670-40676 